\title{
Preparation of manual movements in hemiparkinsonism
}

\author{
ROBERT D RAFAL, JOSEPH H FRIEDMAN, MARGARET C LANNON \\ From the Department of Clinical Neurosciences, Roger Williams General Hospital and Brown University \\ Program in Medicine, Providence, Rhode Island, USA
}

SUMmaRY Twenty patients with asymmetric Parkinson's disease were studied in a reaction time (RT) experiment in which the performance of the more affected ("bad") hand was compared with performance of the less affected ("good") hand. Simple RT and choice RT were tested in separate blocks, and the benefit afforded by advance information in the simple RT condition (choice RT minus simple RT) served as a measure of motor preparation. RT was longer in the "bad" hand in both the simple RT and choice RT conditions. There was no difference in the effect of advance information between the two hands. It is concluded that slowness in RT movement initiation in Parkinson's disease is not due to a deficiency in motor preparation, and that intact basal ganglia function is not required for this stage of motor programming.

The slowing of movement initiation, or reaction time (RT), in Parkinson's disease suggests that Parkinsonians may be deficient in some early stage of motor preparation which precedes movement onset, and that the basal ganglia may function in the preprogramming of movement. One way in which the preparatory stage of motor programming can be measured experimentally employs a movement precuing paradigm. ${ }^{1}$ In this type of task the subject must make one of two (or more) possible movements when a reaction signal is presented. Reaction time is measured under conditions in which advance information is given to the subject which enables him or her to prepare the movement before the reaction signal (simple RT condition); and under conditions where no advance information is available to select the movement prior to the reaction signal (choice RT condition). The advantage afforded by advance information (choice RT minus simple RT) may then serve as an index of motor preparation. Thus, a clinical disorder which disrupts motor programming will permit less benefit from advance information, and should result in a smaller difference between choice RT and simple RT.

Several investigators have now reported that Parkinsonians benefit less from advance information in this type of simple RT $v$ choice RT task ${ }^{2-5}$ than do normal controls. In each of these studies Parkinsonians did Address for reprint requests: Dr D Rafal, Division of Neurology, Roger Williams General Hospital, 825 Chalkstone Avenue, Providence, RI 02908, USA.

Received 15 April 1988 and in revised form 28 October 1988. Accepted 28 October 1988 not differ significantly from controls in choice $\mathrm{RT}$, but were slower under simple RT conditions. These results seem to indicate that slowness in movement initiation in Parkinson's disease results from a defect in motor pre-programming. However, two other investigations using different movement precuing techniques found no defect in motor pre-programming; ${ }^{67}$ and another recent study did not find that the difference between simple RT and choice RT was less in Parkinsonians. ${ }^{8}$ Finally, Rafal et $a l^{13}$ showed that, although simple RT to initiate movement sequences was prolonged in Parkinsonians compared with normal controls, this impairment was not caused by any deficiency in using advance information to program the sequence.

Furthermore, it should be appreciated that the normal advantage for simple RT compared with choice RT does not derive only from motor programming. The precue not only permits the preparation of a motor set, but also establishes a perceptual set; that is, it informs the subject what the reaction signal will look (or sound or feel) like, and it could facilitate its perceptual processing. Thus, the relative deficiency in simple RT reported for Parkinsonians compared with control subjects ${ }^{2-5}$ could reflect difference in cognitive or perceptual abilities between patient and normal populations, rather than a specific deficit in motor preparation.

In the current investigation we sought to determine whether the slowness in initiating reacting time movements in Parkinson's disease is attributable to a deficiency in using advance information in preparing manual movements. We measured simple RT and 
choice RT in patients with asymmetric Parkinsonism in whom one hand was more clinically affected than the other. In this circumstance each subject serves as his or her own control, so that potentially confounding variables such as cognitive, attentional or perceptual deficits are obviated. If slowness in movement initiation in Parkinson's disease is due to defective motor preprogramming, we would expect that slowing of reaction time in the more affected hand would be accompanied by less advantage from information and, therefore, a smaller difference between simple RT and choice RT in the more affected hand.

\section{Subjects and methods}

\section{Subjects}

Twenty patients with idiopathic Parkinson's disease volunteered to participate. They were selected based upon the presence of clear clinical asymmetry of Parkinsonian signs and symptoms. There were six women and 14 men ranging in age from 34-78 yr (mean 60). All were right handed except for one of the left hemiparkisonian subjects. All were active and independent and none had any evidence of dementia on the Mini-Mental State Examination.' Fifteen were taking no conventional anti-parkinsonian medication. Each patient unambiguously identified one hand as his or her "bad" hand. In 15 the other hand was felt to be entirely normal by both patient and examiner. Of these patients, Parkinsonian signs were entirely restricted to the "bad" hand in nine; whereas in six some axial involvement was also present. In the remaining five patients bilateral Parkinsonian signs were present, although in each case a clear asymmetry was evident to both patient and examiner. Although there was bilateral involvement in some patients, we will hereafter designate one hand as the "bad" hand and the other as the "good" hand. Thirteen patients were thus classified as right hemiparkinson and seven were classified as left hemiparkinson.

\section{Apparatus and Procedure}

Subjects sat in a dimly lit room facing a $12^{\prime \prime}$ TV screen display. With one hand they held a lever placed on the table between them and the display. Their task was to move the lever to left or right in response to the appearance of a large, bright arrowhead $(<$ or $>$ ) presented in the centre of the display. The manipulandum was a light lever, constructed from a wooden dowel, which projected vertically from a wooden box. The wrist and forearm rested on the box while the hand held the lever. Within the box the lever was connected to two microswitches. Gentle pressure to right or left activated the switches to record RT. Light pressure moving the lever $2 \mathrm{~mm}$ was sufficient to activate the microswitches in either direction. A strip of light sheet metal under the lever acted as a spring to re-centre the lever after each movement. The display screen and manipulandum were interfaced with an Apple II microcomputer which controlled the display and recorded RT responses on line.

Subjects were instructed to move the lever as quickly as possible in the direction indicated by a large arrowhead presented in the centre of the display. On each trial this reaction signal to "go" pointed, randomly and with equal probability, to left or to right. Each trial began, after an intertrial interval of $1500 \mathrm{~ms}$, with the presentation of a precue which remained visible for $300 \mathrm{~ms}$. In one block, the simple $\mathrm{RT}$ condition, the precue was a small arrowhead which gave advance information of the required movement. In this condition the reaction signal always pointed in the direction indicated by the precue. In another block, the choice RT condition, the precue was a small diamond (constructed of small arrowheads pointing in opposite directions) which alerted the subject, but which provided no information concerning the required direction of the forthcoming movement.

In either condition subjects were cautioned to wait for the reaction signal and not to "jump the gun" when the precue appeared. To discourage time-locked anticipatory responses, the time interval between onset of the precue and appearance of the reaction signal was varied randomly between 750 and $1500 \mathrm{~ms}$. If subjects responded before the reaction signal, or within $100 \mathrm{~ms}$ after it, the response was recorded as an anticipatory error in a feedback signal, "TOO SOON!", was displayed for 2 seconds. The reaction signal remained visible until the subject responded. If no response was made within $5000 \mathrm{~ms}$ a feedback signal "TOO LATE!", was displayed.

Each subject was tested in one session consisting of four blocks; simple RT and choice RT with both the "good" hand and the "bad" hand. Each block consisted of 40 trials and was preceded by 15 practice trials. Both RT conditions were tested in one hand before the other hand was tested. The order of condition (choice RT or simple RT) and hand ("good" hand and "bad" hand) were alternated, independently, across successive subjects.

Three aspects of the data were analysed: anticipatory errors; errors in the direction of movement; and RT for correct responses. For each a $2 \times 2$ repeated measures analysis of variance (ANOVA) was employed with two within factors: Hand ("good" hand or "bad" hand); RT Condition (choice RT or simple RT).

\section{Results}

There were very few anticipatory errors. Anticipatory errors occurred, as expected, more frequently in the simple RT condition $(1.8 \%)$ than in the choice RT condition $(0.3 \%),(F[1,19]=11.2, p<0.005)$. There was no difference in the frequency of anticipatory errors between the two hands. Errors in the direction of movement were also very rare $(0.5 \%)$ and did not differ between the two reaction time conditions, or between the two hands.

Median RT for each subject, for each RT condition and hand was calculated and analysed in an ANOVA. Responses were faster with the "good" hand (F[1,19] $=18.0, \mathrm{p}<0.001)$ and in the simple $\mathrm{RT}$ condition $(\mathrm{F}[1,19]=50 \cdot 3, \mathrm{p}<0.001)$. The table shows the mean RTs for the "good" hand and "bad" hand in each of the two RT conditions. The advantage conferred by the availability of advance information in the simple RT condition (choice RT minus simple RT) was not significantly different between the two hands $(F[1,19]$ 
Table Mean reaction times

\begin{tabular}{lll}
\hline & “Good" Hand & “Bad" Hand \\
\hline Simple RT & 436 & 524 \\
& $(108)$ & $(138)$ \\
Choice RT & 553 & 628 \\
& $(92)$ & $(153)$ \\
\hline
\end{tabular}

$\mathrm{RT}$ in ms. Standard deviations are shown in parentheses.

$=0.78)$. Separate post hoc ANOVAs were made of the simple RT and choice RT data. Mean RT was significantly longer for the "bad" hand in both the simple RT $(F[1,19]=24 \cdot 2, p<0.001)$ and choice $R T$ $(\mathrm{F}[1,19]=10.5, \mathrm{p}<0.005)$ conditions.

\section{Discussion}

In this experiment the more impaired hand of hemiparkinson patients was slower in initiating movements under both simple and choice RT conditions. Moreover, simple and choice RT were slowed to a comparable degree. It should be emphasised here that the more affected hand is not being compared with normal hand function. It is known that greater than $80 \%$ of neuronal function must be lost from substantia nigra before clinical impairment is evident. ${ }^{10}$ Therefore, even in our subjects in whom the "good" hand was clinically intact, we should assume that some physiological dysfunction was present. It was not our intent to compare Parkinsonian with normal function. Rather, we wished to avoid cognitive and attention variables, and to examine specifically the relationship between akinesia in initiating movement and the capacity to use advance information for motor programming. We found that slowing of movement initiation in the more affected hand was not accompanied by a relative deficiency in using advance information in the simple RT condition. Thus, the slowing of movement initiation could not be attributed to an inability to use advance information for motor preparation. The current findings do not support the hypothesis that the basal ganglia play a specific role in the preparatory stage of motor programming which precedes the onset of movement.

Our results are consistent with two previous movement precuing studies in which Parkinsonians were shown to benefit from advance information. In one study Parkinsonians were compared with normal controls; ${ }^{7}$ in the other study patients served as their own controls and their performance compared when on or off treatment. ${ }^{6}$ However, in four other previous studies in which simple and choice RT differences were compared in Parkinsonians and normal control subjects, ${ }^{2-5}$ the difference between these two conditions has been found to be smaller in Parkinsonians; simple RT performance was relatively more impaired in the
Parkinsonians, while choice RT seemed relatively preserved compared with normal controls.

How are we to consider the results of the four studies which found relatively greater impairment in simple RT in Parkinson patients compared with normal control subjects? Do these findings reflect a disorder of motor programming? The current results would suggest that this may not be the case. Moreover, our current findings are consistent with previous studies which have found that Parkinsonian akinesia does not affect the ability to use advance information in programming either choice $\mathrm{RT}^{67}$ or simple $\mathrm{RT}^{11}$ movements.

The recent findings of Pullman et al ${ }^{5}$ are of special interest in this regard. They confirmed that their Parkinsonian subjects were relatively slower under simple RT than under choice RT conditions when compared with a normal control group. They found, however, that as their Parkinson patients became slower with decreasing medication effect, the difference between simple and choice RT actually increased. Thus, increasing akinesia was not associated with less benefit from advance information. While both simple and choice RT increased off medication, the increase in simple RT did not achieve statistical significance; whereas the increase in choice RT off medication was significantly greater than simple RT. Pullman et al speculated that the increase in choice $R T$ off medication might reflect a cognitive impairment related to dopamine deficiency. Our observations in hemiparkinsonians, most of whom were unmedicated, indicate however, that Parkinsonian akinesia can slow both simple and choice RT independent of any cognitive factors.

Patients with Parkinson's disease may be relatively more impaired under simple RT than choice RT conditions, at least in some tasks and when compared with normal control subjects. However, the akinesia of Parkinson's disease does also slow choice RT, and does not seem to affect simple RT disproportionately. The reported relative deficiency in simple RT performance in Parkinson's disease patients compared with normal individuals appears to be a phenomenon which is independent of Parkinsonian akinesia, and not the cause of it. The reason for this phenomenon remains unclear. However, it appears to be unlikely that it is due to a defect in motor programming or a cause of akinesia in Parkinson's disease, and the explanation for it might best be sought elsewhere.

This study was supported by grants from the US Public Health Service R01-MH41544 and R01NS24778. We are grateful for assistance from the Rhode Island Parkinson's Disease Referral and Information Center and to Corrine Hopp for manuscript preparation. 


\section{References}

1 Rosenbaum DA. Human movement initiation: Specification of arm, direction and extent. J Exp Psychol [Gen] 1980;109:444-74.

2 Evarts EV, Teravainen H, Calne DB. Reaction time in Parkinson's disease. Brain 1981;104:167-86.

3 Bloxham CA, Mindel TA, Frith CD. Initiation and execution of predictable and unpredictable movements in Parkinson's disease. Brain 1984;107:371-84.

4 Sheridan MR, Flowers KA, Hurrell J. Programming and execution of movement in Parkinson's disease. Brain 1987;110:1247-71.

5 Pullman SL, Watts RL, Juncos JL, Chase TN, Sanes JN. Dopaminergic effects on simple and choice reaction time performance in Parkinson's disease. Neurology 1988;38:249-54.

6 Rafal RD, Posner MI, Walker JA, Friedrich FJ. Cognition and the basal ganglia: Separating mental and motor components of performance in Parkinson's disease. Brain 1984;107:1083-94.

7 Stelmach GE, Worringham CJ, Strand EA. Movement preparation in Parkinson's disease. Brain 1986;109: 1179-94.

8 Mayeux R, Stern Y, Sano M, Cote L, Williams JBW. Clinical and biochemical correlates of bradyphrenia in Parkinson's disease. Neurology 1987;37:1130 and 1693.

9 Folstein MF, Folstein SE, McHugh P. Mini-Mental State: A practical method for grading the cognitive state of patients for the clinician. $J$ Psychiatr Res 1975;12:189-98.

10 Bernheimer H, Birkmayer W, Hornykiewicz O, Jellinger $\mathrm{K}$, Seitelberger F. Brain dopamine and the syndromes of Parkinson and Huntington. J Neurol Sci 1973;20: 415-55.

11 Rafal RD, Inhoff AW, Friedman JH, Bernstein E. Programming and execution of sequential movements in Parkinson's disease. J Neurol Neurosurg Psychiatry 1987;50:1267-73. 